\title{
Superior Structural Quality of Newly Developed GaN Pendeo-Epitaxial Layers
}

\author{
Z. Liliental-Weber, ${ }^{a}$ J. Jasinski, ${ }^{a}$ D. Cherns, ${ }^{b}$ M. Baines, ${ }^{b}$ and R. Davis ${ }^{c}$ \\ ${ }^{a}$ Lawrence Berkeley National Laboratory, Berkeley, CA 94720 m/s 62/203 \\ ${ }^{\mathrm{b}}$ H.H. Wills Physics Laboratory, University of Bristol, Bristol, UNITED KINGDOM \\ ${ }^{\mathrm{c}}$ Department of Materials Science and Engineering, University of North Carolina, Raleigh, NC.
}

\begin{abstract}
:
Transmission electron microscopy of plan-view and cross-section samples of pendeo-epitaxial layers is described. Samples grown with and without silicon nitride masks are compared. A large misorientation of the $\mathrm{GaN}$ grown above the mask was observed, with $2-3^{\circ}$ tilt between wing and seed areas, caused by additional nucleation on the mask layer. Some misorientation was also observed between wing/wing areas of the sample. Samples grown without silicon nitride masks show much smaller misorientations and contain different types of defects.
\end{abstract}

\section{INTRODUCTION}

The lack of large, free standing GaN substrates for the growth of homoepitaxial layers is forcing investigators in the III- nitride community to develop special growth methods in order to decrease dislocation density in heteroepitaxially grown material, used for different device applications. Two related methods: laterally epitaxial overgrowth (LEO) [1-4] and pendeoepitaxy (PE) have been developed [5-8]. Application of both these methods leads to a much lower density of dislocations, especially those that propagate in the growth direction. In the pendeo-epitaxy method, growth does not initiate through the open windows on the (0001) as it does in LEO structures, but instead it is forced to begin on the sidewalls etched into the seed crystal to form overhanging wings. All layers studied in this paper were grown on $\mathrm{SiC}$ with an AlN buffer layer, which had then been selectively etched; etching continued into the $\mathrm{SiC}$. The details of the growth procedure are described in several previous papers [5,7]. Here the microstructure of two (masked and unmasked) PE structures will be described in detail.

\section{EXPERIMENTAL}

Masked PE structures consisted of a $100 \mathrm{~nm}$ silicon nitride layer deposited onto the etched GaN/AlN seeds (about $1.2 \mu \mathrm{m}$ in height). The seeds ( $2 \mu \mathrm{m}$ wide strips) were about $3 \mu \mathrm{m}$ apart. The PE layers coalesced and the total thickness of the layers measured between the seeds was about $2 \mu \mathrm{m}$, with about $1.2 \mu \mathrm{m}$ above the $\mathrm{Si}_{3} \mathrm{~N}_{4}$ mask. In the second sample, which was prepared without a silicon nitride cap, the seed height was only about $0.15 \mu \mathrm{m}$, and the seeds were separated by about $4 \mu \mathrm{m}$. The total PE layer thickness between the seeds was measured to be about $3.5 \mu \mathrm{m}$. Schematic drawings of these samples are shown in Fig. 1. Thin foils, transparent to electrons, were prepared using mechanical polishing (dimpling) and Argon ion-milling, in the cross-section perpendicular to the [1100] seed-stripe direction, and also in plan-view orientation, where the thinning was performed from the substrate in order to obtain information from the area 
close to the sample surface. A Topcon 002B electron microscope with $200 \mathrm{KeV}$ acceleration voltage and Philips 430 microscope operated at $250 \mathrm{KeV}$ with sample tilting of up to about $\pm 60^{\circ}$ were used for these studies. A standard g.b analysis was used to determine dislocation Burgers vectors. Convergent Beam Electron Diffraction (CBED), or Large Angle CBED (LACBED), was used to determine the tilt/twist angles between wing/wing and wing/seed areas.

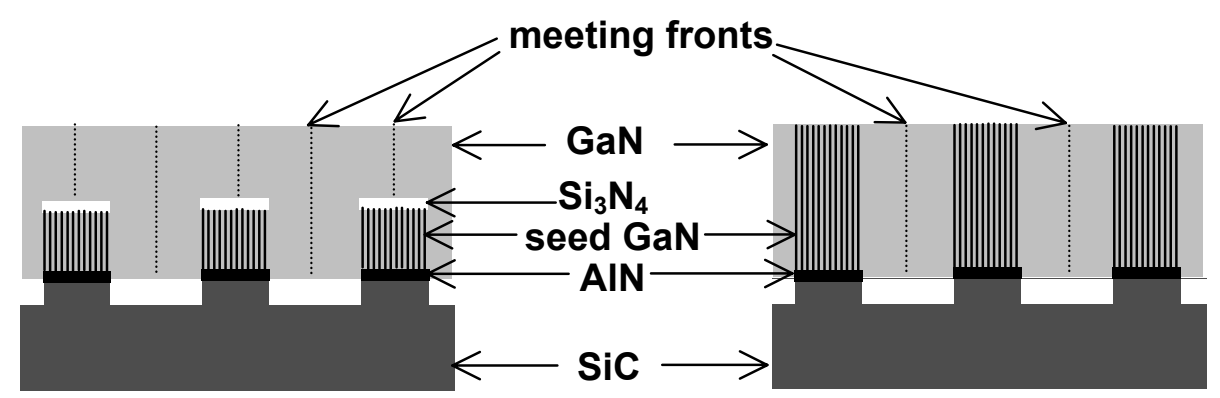

Fig. 1. Schematic diagrams of the pendeo-epitaxial layers grown with (left) and without (right) the silicon nitride mask. Vertical lines indicate dislocations

\section{RESULTS AND DISCUSSION}

Cross-section studies of the PE layers grown with the $\mathrm{Si}_{3} \mathrm{~N}_{4}$ mask show that the layer has grown sideways from the seed (Fig. 2a). A clear gap was observed between the PE layer and the $\mathrm{SiC}$ substrate. Dislocation half-loops are seen to propagate from the seed on the c-planes and their spacing increases with increasing distance from the substrate (Fig. 2b). The distance between these dislocation loops in the area closer to the substrate was about 50-100 nm along the c-direction, but, closer to the top surface, the distance between these defects was more than 500 $\mathrm{nm}$. In the area where two wings meet, voids were formed (Fig. 2c) and usually the defect density below the void (closer to the substrate) was higher than above them. In many cases edge dislocations were formed above the void (closer to the surface) but occasionally defect free material could also be observed, which indicates some non-uniformity of the PE layers grown between the seeds.

It could be observed that the layers were not only growing laterally from the seeds but that growth also occurred above $\mathrm{Si}_{3} \mathrm{~N}_{4}$ masks. Close to the edges of the mask, lateral growth took place, but some misorientation can be observed in these areas. This misorientation increased for the growth over the central parts of the mask. One can notice on Fig. 2a that two clear boundaries were formed in the GaN grown above the mask. At some boundaries small nanotubes (voids) have developed. This observation indicates that some nucleation took place independently of the overgrowth, which can be seen on Fig. 2a where a central grain is separated from the overgrown areas by grain boundaries (arrows). This was also confirmed for PE growth with larger $5 \mu \mathrm{m}$ $\mathrm{Si}_{3} \mathrm{~N}_{4}$ masks, where two or three independent grains were formed above the mask (these results are not shown here due to a lack of space). 


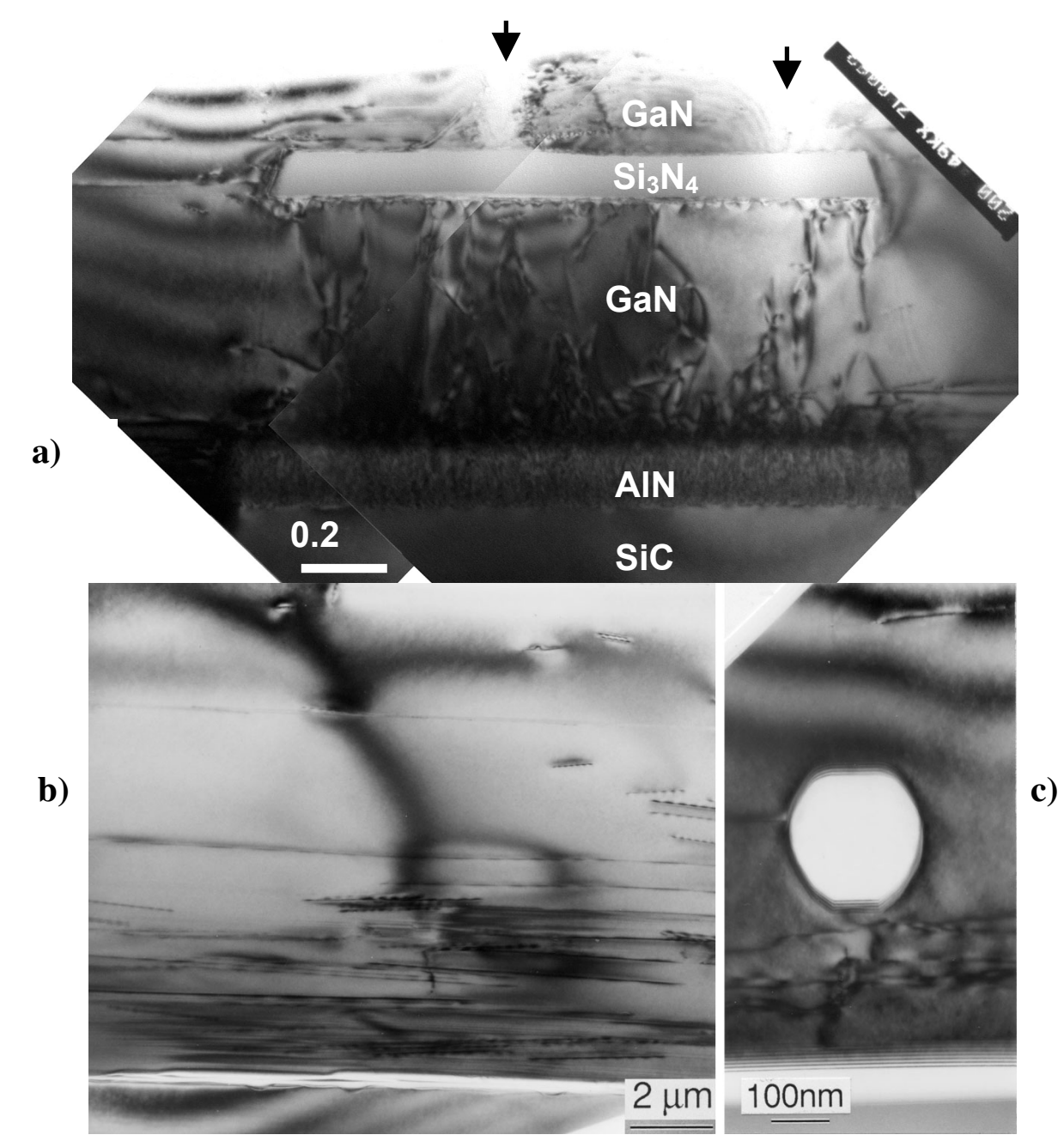

Fig. 2. (a) A micrograph from the cross-section sample grown with the $\mathrm{Si}_{3} \mathrm{~N}_{4}$ mask. Note the clear boundaries between the grains formed in the area above the mask; (b) dislocation loops formed in the wing area. Note their higher density in the area closer to the substrate and the gap formed between the PE layer and the substrate confirming that growth takes place sideways from the seed; (c) The wing/wing meeting front showing formation of a void. Note the different density of defects above and below the void.

Studies of a plan view sample confirmed that there were often misorientations of up to $2-3^{\circ}$ between the seed crystal and the wings, or across the meeting-front between adjacent wings. Figure 3 shows a seed region $B$ and adjacent wings $A$ and $C$, along with the CBED patterns from all three regions. It is clear from the CBED patterns that the wings $\mathrm{A}$ and $\mathrm{C}$ are similarly oriented and that region $\mathrm{B}$ is misoriented from both by roughly $2^{\circ}$ about the $(1100)$ axis. Notice the displacement of the (1120) Kikuchi band (K) relative to the central spot (shown by a circle). This angular displacement is associated with a series of half loops that have opposite segments in the seed/wing boundaries. The Burgers vectors of these segments are in edge orientation, forming the tilt boundaries evident from the CBED patterns. 


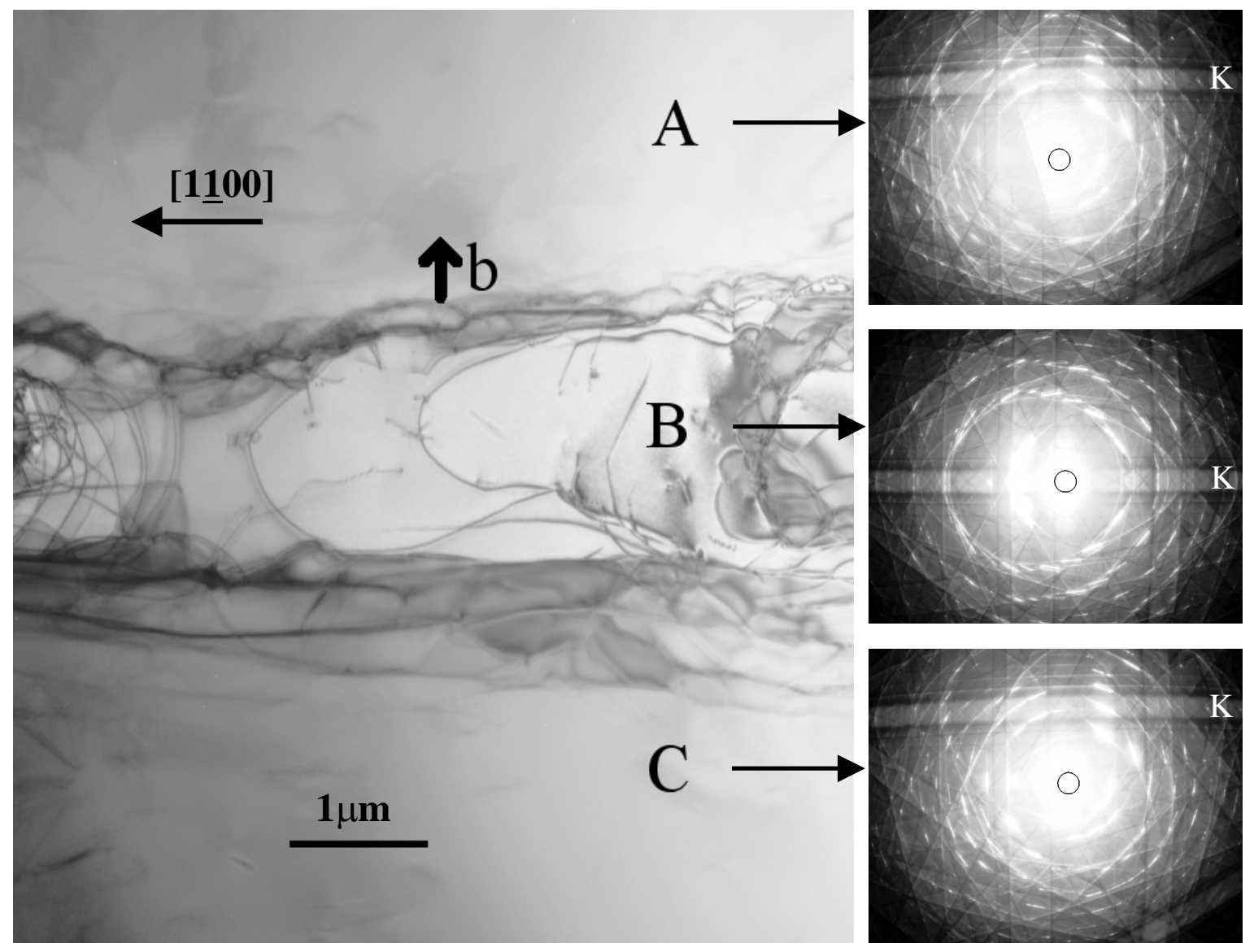

Fig. 3. Plan-view micrograph of the GaN layer grown above the silicon nitride mask (B) and adjacent wings (A and C), with CBED patterns from each region, showing the (1120) Kikuchi band (K). Note the different distance between the central beam (marked by circle) and the Kikuchi band, indicating a misorientation.

Fig. 4a shows a region where adjacent wing/wing crystals are misoriented. This is revealed by a change in diffraction conditions, giving dark contrast on the upper wing and light contrast on the lower wing. It was observed that misorientations across the seed/wing and wing/wing boundaries varied from one region to another, in agreement with the observation from crosssection samples, and were mainly tilts (about an axis parallel to the seed stripe direction) rather than twists. The observations suggested that half-loops were generated to accommodate these tilts.

A substantially different defect arrangement was observed in the samples grown without $\mathrm{Si}_{3} \mathrm{~N}_{4}$ masks. Dislocations that were present in the seeds propagated to the sample surface, as one can see in Fig. 5. In the wings of the PE layers, dislocations were formed on c-planes in the areas close to the substrate, as was observed in the sample with the $\mathrm{Si}_{3} \mathrm{~N}_{4}$ mask, up to about $1 \mu \mathrm{m}$ from the bottom of the PE layer. Large voids were formed at the meeting front of the two wings. All these voids were overgrown and dislocations were formed in this area of the crystal. Analysis of these dislocations showed that most of them had screw character. The distribution of these dislocations on the sample surface can more easily be observed in plan-view orientation, as shown in figure $4 \mathrm{~b}$. 

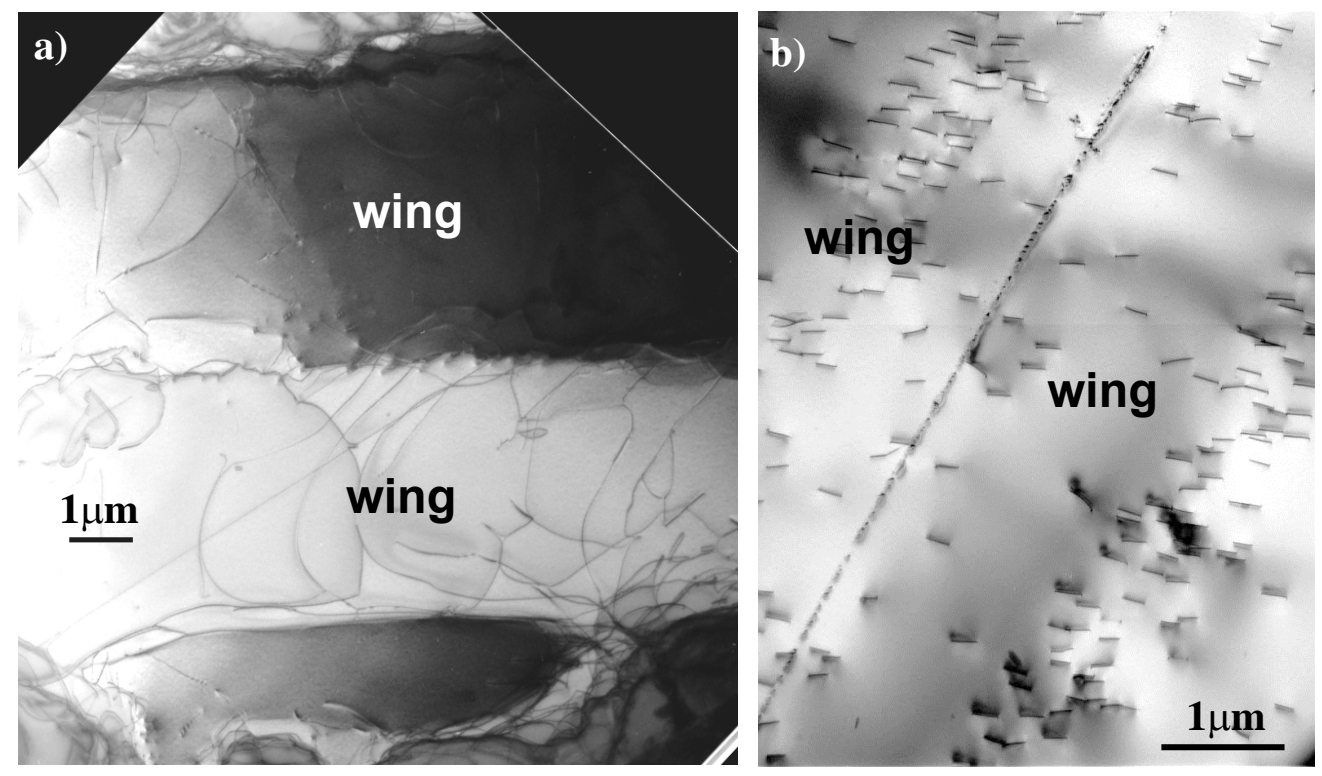

Fig. 4. Bright field micrographs of plan-view samples, (a) with, and (b) without, the silicon nitride mask. (a) shows the wing/wing area with adjacent areas grown over the seeds (extreme top and bottom of picture); note darker contrast in the upper wing indicating some misorientation and formation of half-loops in the wings, with threading dislocations seen edge-on on the meeting front. (b) shows the distribution of defects near a wing-wing boundary.

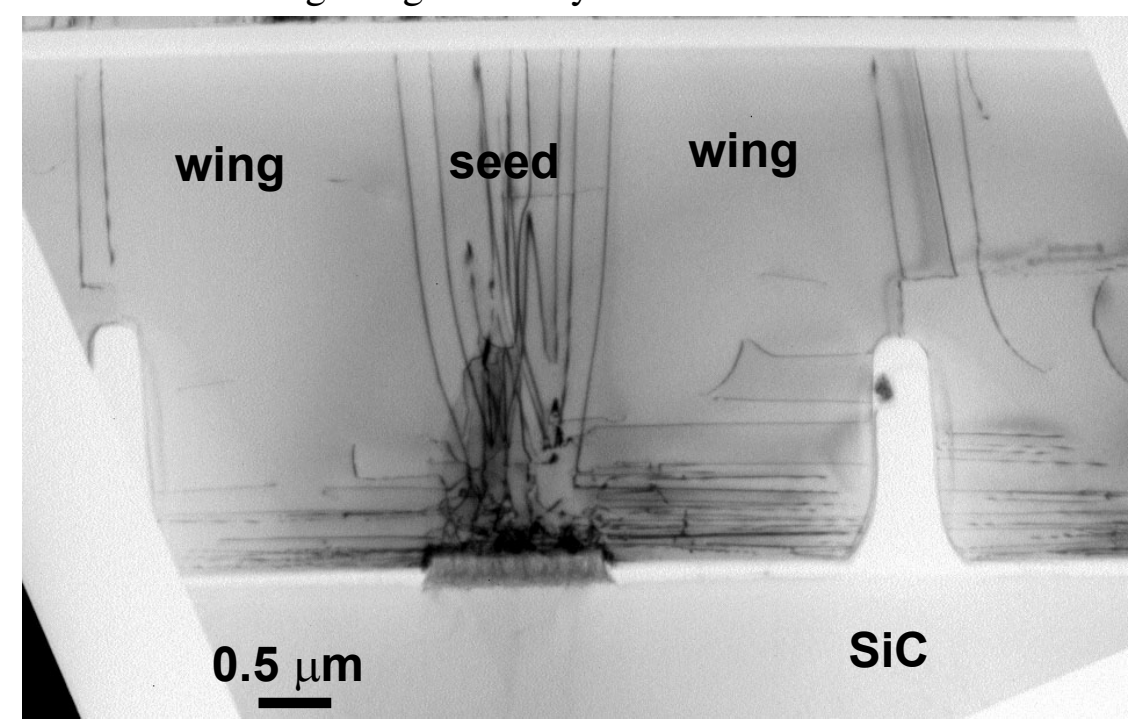

Fig. 5. Cross-section micrograph from the PE sample grown without the mask showing defect distribution over the seed and in the wings. Note the uniform contrast indicating that the misorientation in this sample is small.

In figs 4(b) and 5, one can notice that only one meeting front (MF) was formed, in the wing/wing area, and that there is no additional MF in the areas over the seeds. The density of dislocations in the wing areas was much smaller $\left(1.2 \times 10^{8} \mathrm{~cm}^{-2}\right)$ than in the areas grown over the seeds $\left(1.5 \times 10^{9} \mathrm{~cm}^{-2}\right)$. It appeared that such samples were more uniform; no contrast difference 
was noticed between different sides of the wing/wing MF. One can also notice that the type of dislocations in the overgrown area is different in the samples grown with and without the mask. In the latter sample, more threading dislocations (similar to the dislocations formed in the areas grown over the seed) can be observed. These are present instead of the half loops formed in the PE samples grown with the $\mathrm{Si}_{3} \mathrm{~N}_{4}$ mask, or observed in samples laterally overgrown $[3,4]$.

From Fig. 5 one can notice that the contrast in the wings on either side of the seed is practically identical, but there are areas where some small differences could be observed. CBED patterns (not shown for lack of space) taken in the areas where some difference in contrast across the wing/wing MF was evident, indicate a misorientation of about $0.15^{\circ}$ twist. Similar twist misorientation can, occasionally, also be observed at the seed-wing interface; however this twist is not uniform, and there are some regions where no twist (and no dislocation) is present. CBED analysis also suggests that the sample was uniformly curved; about $1^{\circ}$ tilt per 20 microns can be observed.

In summary, we compared two different pendeo-epitaxial samples grown with and without a $\mathrm{Si}_{3} \mathrm{~N}_{4}$ mask, and found different types and distributions of defects. We also showed that in the samples with the mask, additional nucleation of the $\mathrm{GaN}$ is taking place and that additional grain boundaries can be formed in the area grown over the mask, leading to a large tilt (up to 2$3^{\circ}$ ) between the seed and wing areas. The sample grown without the mask is more uniform, and only very minor twist in some areas of the sample can be detected using sensitive CBED measurements.

\section{ACKNOWLEDGMENTS:}

This research was supported by Air Force Office of Scientific Research, through the U.S. Department of Energy under Order No. AFOSR-ISSA-00-0011. TEM sample preparation by W. Swider and use of facilities in NCEM LBNL and Bristol University are greatly appreciated.

\section{REFERENCES:}

1. H. Marchand, J.P. Ibbetson, P.T. Fini, P. Kosodoy, S. Keller, S. DenBaars, J.S. Speck, U.K. Mishra, MRS Internet J. Nitride Semicond. Res. 3, 3 (1998).

2. A. Sakai, H. Sunakawa, A. Usui, Appl. Phys. Lett. 71, 2259 (1997).

3. Z. Liliental-Weber, M. Benamara, W. Swider, J. Washburn, J. Park, P. A. Grudowski, C. J. Eiting, and R. D. Dupuis, MRS Internet J. Nitride Semicond. Res. Res. 4S1, G4.6, (1998).

4. Z. Liliental-Weber and D. Cherns, J. Appl. Phys. 89, 7833 (2001).

5. T. Zheleva, S. Smith, D. Thomson, K. Linthicum, P. Rajagopal, R.F. Davis, J. Electr. Mat. 28 (4), L5 (1999).

6. T. Zheleva, S. Smith, D. Thomson, K. Linthicum, P. Rajagopal,, E. Carlson, W. Ashmawi, and R.F. Davis, MRS Internet J. Nitride Semicond. Res. 4S1, G49 (1999).

7. P. Fini, H. Marchand, J.P. Ibbetson, B. Moran, L. Zhao, S.P. DenBaars, J.S. Speck, U. Mishra Mater. Res. Soc. Symp. Proc. 572, 315 (1999).

8. R.F. Davis, T. Gehrke, K.J. Linthicum, T.S. Zhelewa. E.A. Preble, P. Rajagopal, C.A. Zorman, and M. Mehregany, J. Crystal Growth 225, 134 (2001). 